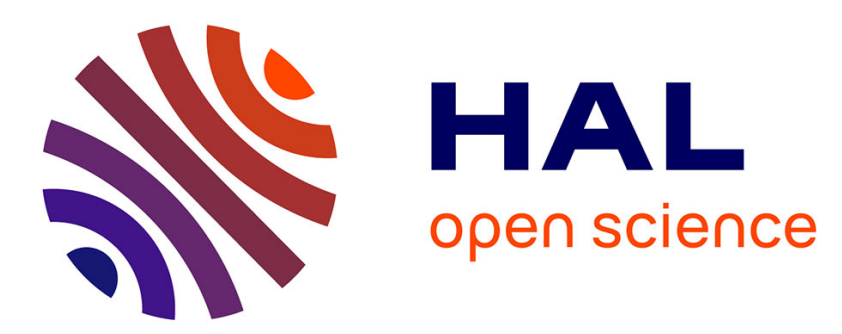

\title{
Étude comparative des fonctionnalités des moteurs de recherche d'images sur Internet
}

Christophe Boudry, Clémence Agostini

\section{To cite this version:}

Christophe Boudry, Clémence Agostini. Étude comparative des fonctionnalités des moteurs de recherche d'images sur Internet. Documentaliste - Sciences de l'Information, 2004, 41 (2), pp.96-105. hal-00596119

\section{HAL Id: hal-00596119 \\ https://hal.science/hal-00596119}

Submitted on 26 May 2011

HAL is a multi-disciplinary open access archive for the deposit and dissemination of scientific research documents, whether they are published or not. The documents may come from teaching and research institutions in France or abroad, or from public or private research centers.
L'archive ouverte pluridisciplinaire HAL, est destinée au dépôt et à la diffusion de documents scientifiques de niveau recherche, publiés ou non, émanant des établissements d'enseignement et de recherche français ou étrangers, des laboratoires publics ou privés. 


\title{
Étude comparative des fonctionnalités des moteurs de recherche d'images sur Internet
}

\author{
Par CHRISTOPHE BOUDRY, URFIST de Paris, et CLEMENCE AGOSTINI
}

Le réseau Internet offre un gisement d'images numériques considérable [1]. La localisation de ces images passe le plus souvent par l'utilisation d'outils de recherche spécialement dédiés à la recherche d'images. Si la littérature est assez riche en ce qui concerne les outils de recherche de pages web [2-7], peu d'articles ou d'études se sont attachés jusqu'à présent à décrire le fonctionnement des outils de recherche d'images [8-15]. L'objectif de cet article est donc de comparer les fonctionnalités de ces outils de recherche, en distinguant ceux qui dérivent directement d'outils de recherche de pages web de ceux qui ont été spécialement créés et développés pour la recherche d'images sur Internet.

\section{Note sur les auteurs}

\section{Christophe Boudry}

est maître de conférences à l'Urfist de Paris / École nationale des chartes (17 rue des Bernardins, F-75005 Paris, téléphone +33 (0)1 432685 22, courriel boudry@ccr.jussieu.fr) et au Groupe Régional d'Etudes sur le Cancer (GRECAN EA-1772).

\section{Clémence Agostini}

est diplômée du cycle supérieur de l'Institut national des techniques de la documentation (INTD) et actuellement consultante chez Locarchives.

\section{Rappels sur l'indexation et les méthodes de recherche d'images}

Il existe deux types de méthodes d'indexation et de recherche d'images: par le texte et par l'image.

\section{Recherche par le texte}

La première, dénommée le plus souvent «concept-based image retrieval» dans la littérature anglo-saxonne, est actuellement la plus utilisée.

Paradoxalement, elle n'utilise pas les données des images lors du processus d'indexation et de recherche, mais des éléments textuels extra-picturaux. Dans le cas d'images présentes dans des collections, l'indexation s'effectue « manuellement » : l'indexeur crée une notice qui décrit l'image 
grâce à des éléments textuels. Les difficultés rencontrées lors de ce processus sont nombreuses: diversité des niveaux de description et d'interprétation des images (appréhension dénotative ou connotative de celles-ci), polysémie, manque de reproductibilité inter-indexeur, coût en temps, problème de l'adéquation de l'indexation avec les aspirations des utilisateurs de la collection d'images, évolution du langage d'indexation avec le temps. L'analyse de ces difficultés a fait l'objet de nombreux ouvrages et articles. [16-24].

Dans le cas des images présentes sur les sites Internet, l'indexation par les outils de recherche d'images est totalement automatique, sans intervention humaine. Le repérage des images s'effectue grâce à leur extension et le texte indexé avec l'image est extrait du code HTML de la page support de l'image. Il s'agit le plus souvent du contenu de la balise SRC (qui contient le nom du fichier image), du contenu de la balise ALT de l'image (pas toujours renseignée, mais qui est censée fournir une description de l'image), du titre de la page web, ou bien encore du texte «proche» de l'image présent sur la page web [11, 15]. La phase de recherche d'images par l'usager s'apparente dans tous les cas à une recherche textuelle « classique ».

\section{Recherche par l'image}

Les méthodes de recherche par l'image, dénommées content-based image retrieval (CBIR) dans la littérature anglo-saxonne, sont par comparaison beaucoup plus récentes (le terme CBIR est apparu pour la première fois en 1992 [25]).

Avec ce type de méthodes, la phase d'indexation des documents n'implique à aucun moment l'utilisation du langage. Elle consiste à extraire un certain nombre (xpar exemple) de paramètres d'analyse d'images (paramètres de couleur, texture, intensité ou forme) [26-28], qui sont à même de caractériser de manière discriminante les images présentes dans la base de données. Chaque image est alors identifiée non pas par des éléments textuels, mais par les valeurs de ses $x$ paramètres (on parle également de signatures). Chaque image est alors «positionnée 》 dans un espace de référence en fonction de la valeur de ses x paramètres.

Le processus de recherche s'effectue non pas à partir d'une requête textuelle, mais à partir d'une image "requête» pour laquelle les $x$ paramètres sont calculés. L'image requête est alors "placée» dans l'espace de référence où sont présentes les images indexées. Les images les plus proches de l'image requête dans cet espace de référence (et ayant donc des valeurs paramétriques similaires), sont alors présentées en résultat à l'utilisateur comme étant les images les plus proches visuellement de l'image requête.

Les avantages de ces méthodes par rapport aux méthodes de recherche d'images par le texte sont de plusieurs types. De par leur mode de fonctionnement, elles nobligent pas à conceptualiser la recherche avec des mots et s'affranchissent donc des difficultés liées au langage rencontrées avec les premières. Ces méthodes permettent en outre de répondre à des questionnements ne faisant pas appel à un cadre sémantique (en permettant par exemple de rechercher des images contenant certaines gammes de couleurs ou d'effectuer une recherche à partir d'un exemple d'image existant ou créée pour l'occasion).

Les difficultés rencontrées avec ces méthodes sont principalement liées au fait qu'il n'est pas forcément aisé de rendre compte de la perception visuelle par des paramètres d'analyse d'images. Il peut ainsi exister un certain déphasage entre ce que la méthode de recherche par l'image considère comme des images similaires d'un point de vue paramétrique et ce que l'œil de l'utilisateur considère comme des images similaires d'un point de vue visuel (c'est particulièrement le cas en ce qui concerne la texture des images [28]). De plus, ces méthodes sont relativement complexes à mettre au point et nécessitent un certain savoir-faire en 
traitement et analyse d'images. Pour maximiser les performances de ces méthodes, il faut en effet trouver des paramètres discriminants pour différencier les images et, si possible, en petit nombre pour faciliter les traitements ultérieurs. Si cela est souvent possible lorsque les images sont de même type et contiennent des invariants (image de visage, d'empreintes digitales, etc.), il est en revanche très difficile de trouver une combinaison de paramètres discriminants pour des images dont le contenu varie grandement (ce qui est évidemment le cas pour les images présentes sur Internet).

Enfin, si les méthodes de recherche d'images par le texte permettent de répondre à des interrogations de bas niveau (recherche d'objets, personnes, etc.) ou de niveau plus élevé (recherche d'attributs abstraits des images), les méthodes de recherche par l'image permettent en revanche de répondre presque uniquement à des interrogations de bas niveau concernant les caractéristiques primitives des images (forme, intensité, texture, couleurs) ou, au mieux, la reconnaissance d'objets d'un type donné.

Toutes ces difficultés expliquent pourquoi ces méthodes de recherche par l'image n'ont été jusqu'à présent implémentées avec succès que sur des bases de données d'images homogènes dans des domaines précis, comme ceux de la criminologie (par exemple dans la recherche de visages [30]) ou de la propriété intellectuelle [31]. Seuls deux outils de recherche d'images sur Internet ont à l'heure actuelle mis en cuvre ce type de méthodes: Altavista et Yahoo!, respectivement avec leurs interfaces Altavista Photo Finder et Image Surfer en collaboration avec Virage (VIR Image Engine) et Excalibur (Excalibur Visual RetrievalWare). Les faibles performances obtenues ainsi ont, entre autres, contraint les concepteurs de ces deux outils à faire machine arrière et à ne plus proposer ce type de recherche. (À noter que ces deux outils permettaient de rechercher par l'image exclusivement à la suite d'une première requête textuelle, et ceci uniquement sur certaines images.)

Actuellement, les seuls outils de recherche d'images sur Internet utilisant les méthodes de recherche par l'image en sont encore au stade expérimental [32, 33].

\section{Les outils de recherche étudiés}

Le groupe d'outils étudié est composé de sept moteurs de recherche d'images: AllTheWeb (www.alltheweb.com), Altavista (www.altavista.com), Google (www.google.com), Lycos (multimedia.lycos.com), Picsearch (www.picsearch.com), Ditto (www.ditto.com) et Visoo (www.visoo.com).

Les quatre premiers dérivent de moteurs généralistes qui ont développé une interface permettant de rechercher des images en plus de la recherche de pages web qu'ils proposaient antérieurement. Ainsi, Lycos et Al/TheWeb existent respectivement depuis juin 1995 et mai 1999. Le moteur de recherche de pages web Altavista, créé le 15 décembre 1995, propose une interface pour rechercher des images depuis l'automne 1998. Google, enfin, a été lancé en 1998 et propose une recherche d'images depuis juin 2001.

Trois de ces moteurs, AllTheWeb, Altavista et Lycos, permettent également de rechercher des sons et des vidéos. Ces options peuvent ainsi être regroupées dans une catégorie globale de recherche multimédia, comme c'est le cas pour Lycos. Google, au contraire, propose pour l'instant uniquement la recherche d'images.

Les trois moteurs restants, Ditto, Picsearch et Visoo, ont été spécialement développés pour la recherche d'images et ne proposent pas d'autres possibilités de recherche. Ditto a été créé le 
10 novembre 1998 sous le nom d'Arribavista, alors que Picsearch n'a vu le jour que le 2 août 2001, soit deux mois après l'apparition de l'interface image de Google. Visoo est quant à lui le plus récent de tous ces outils (à noter qu'il n'est plus fonctionnel au moment de la rédaction de cet article).

\section{Comparaison des langages et syntaxes d'interrogation}

L'objet de cette partie est de comparer les possibilités offertes par chaque outil sur le plan de la formulation syntaxique des requêtes.

Le tableau 1 regroupe à gauche les quatre moteurs généralistes, Al/TheWeb, Altavista, Google et Lycos, et à droite les trois outils spécialisés dans la recherche d'images, Ditto, Picsearch et Visoo. Quinze fonctionnalités ont été examinées: les opérateurs syntaxiques que l'utilisateur peut employer (AND, OR, AND NOT, +, -, NEAR), l'opérateur effectif par défaut, la possibilité de faire une équation de recherche avec des parenthèses, de rechercher une expression exacte, de poser une requête avec troncature, la sensibilité de l'outil à la casse, le respect des accents, l'existence d'un filtre de contenu, la possibilité de limiter sa recherche par certains critères (la langue, la date, le site, etc.) ou sur un champ particulier (le titre de la page, l'URL, etc.), et enfin la possibilité d'affiner sa recherche. Chacun de ces critères (comme d'ailleurs tous ceux qui sont étudiés dans cet article) a été testé en pratique sur chaque outil de recherche pour déterminer de manière expérimentale s'ils étaient réellement disponibles et fonctionnels sur chacune des interfaces. 


\begin{tabular}{|c|c|c|c|c|c|c|c|}
\hline & $\begin{array}{l}\text { AllThe } \\
\text { Web }\end{array}$ & Altavista & Google & Lycos & Ditto & Picsearch & Visoo \\
\hline $\begin{array}{l}\text { opérateur } \\
\text { par défaut }\end{array}$ & AND & OR & AND & AND & OR & AND & OR \\
\hline ET & défaut & $\mathrm{O}$ & défaut & défaut & $\mathrm{N}$ & défaut & $\mathrm{N}$ \\
\hline OU & $\mathrm{O}$ & défaut & $\mathrm{O}$ & $\mathrm{N}$ & défaut & $\mathrm{N}$ & $\mathrm{O}$ \\
\hline SAUF & $\mathrm{N}$ & $\mathrm{O}$ & $\mathrm{N}$ & $\mathrm{N}$ & $\mathrm{N}$ & $\mathrm{O}$ & $\mathrm{N}$ \\
\hline opérateur + & $\mathrm{O}$ & $\mathrm{O}$ & $\mathrm{O}$ & $\mathrm{O}$ & $\mathrm{N}$ & $\mathrm{O}$ & $\mathrm{O}$ \\
\hline opérateur - & $\mathrm{O}$ & $\mathrm{O}$ & $\mathrm{O}$ & $\mathrm{O}$ & $\mathrm{N}$ & $\mathrm{O}$ & $\mathrm{O}$ \\
\hline troncature & $\mathrm{N}$ & $\mathrm{O}$ & $\mathrm{N}$ & $\mathrm{N}$ & $\mathrm{N}$ & $\mathrm{N}$ & $\mathrm{N}$ \\
\hline expression & $\mathrm{O}$ & $\mathrm{O}$ & $\mathrm{O}$ & $\mathrm{O}$ & $\mathrm{N}$ & $\mathrm{N}$ & $\mathrm{N}$ \\
\hline proximité & $\mathrm{N}$ & $\mathrm{O}$ & $\mathrm{N}$ & $\mathrm{N}$ & $\mathrm{N}$ & $\mathrm{N}$ & $\mathrm{N}$ \\
\hline parenthèses & $\mathrm{N}$ & $\mathrm{O}$ & $\mathrm{N}$ & $\mathrm{O}$ & $\mathrm{N}$ & $\mathrm{N}$ & $\mathrm{N}$ \\
\hline casse & $\mathrm{N}$ & $\mathrm{N}$ & $\mathrm{N}$ & $\mathrm{N}$ & $\mathrm{N}$ & $\mathrm{N}$ & $\mathrm{N}$ \\
\hline accents & $\mathrm{O}$ & $\mathrm{O}$ & $\mathrm{N}$ & $\mathrm{O}$ & $\mathrm{N}$ & $\mathrm{O}$ & $\mathrm{N}$ \\
\hline$\left|\begin{array}{ll}\text { filtre } & \text { de } \\
\text { contenu }\end{array}\right|$ & $\begin{array}{l}\text { O par } \\
\text { défaut }\end{array}$ & $\begin{array}{l}\text { O par } \\
\text { défaut }\end{array}$ & $\begin{array}{l}\text { O par } \\
\text { défaut }\end{array}$ & $\begin{array}{c}\text { O à } \\
\text { installer }\end{array}$ & O obligatoire & O obligatoire & $\begin{array}{l}\text { O par } \\
\text { défaut }\end{array}$ \\
\hline$\left|\begin{array}{l}\text { limiter la } \\
\text { recherche }\end{array}\right|$ & $\mathrm{N}$ & $\begin{array}{c}\text { nom de } \\
\text { domaine, } \\
\text { host }\end{array}$ & $\begin{array}{l}\text { nom de } \\
\text { domaine, } \\
\text { site }\end{array}$ & $\mathrm{N}$ & $\mathrm{N}$ & $\mathrm{N}$ & $\mathrm{N}$ \\
\hline $\begin{array}{l}\text { recherche } \\
\text { sur des } \\
\text { champs }\end{array}$ & $\mathrm{N}$ & title, url & $\mathrm{N}$ & $\mathrm{N}$ & $\mathrm{N}$ & $\mathrm{N}$ & $\mathrm{N}$ \\
\hline $\begin{array}{l}\text { raffiner la } \\
\text { recherche }\end{array}$ & $\mathrm{N}$ & $\mathrm{O}$ & $\mathrm{N}$ & $\mathrm{N}$ & $\mathrm{N}$ & $\mathrm{N}$ & $\mathrm{N}$ \\
\hline Total & 7 & 13 & 8 & 7 & 3 & 6 & 4 \\
\hline Moyenne & \multicolumn{4}{|c|}{8,75} & \multicolumn{3}{|c|}{4,33} \\
\hline
\end{tabular}

Tableau 1: syntaxe de recherche des 7 outils de recherche d'images étudiés. $N$ : fonctionnalité non disponible pour l'outil considéré : $O$ : fonctionnalité disponible pour l'outil considéré.

\section{Caractéristiques des moteurs généralistes}

Concernant l'interface image des quatre moteurs généralistes, on peut observer qu'Altavista est de loin celui qui propose la plus grande quantité d'options (treize sur quinze). Il s'agit du seul moteur à permettre de formuler une équation avec AND NOT (mais les autres permettent d'utiliser le signe - qui est équivalent), de faire usage de la troncature, ainsi que de l'opérateur de proximité NEAR. Par ailleurs, Altavista est le seul de ces quatre moteurs dont l'opérateur par défaut est OR. 
Les trois autres moteurs généralistes, AllTheWeb, Google et Lycos, comptabilisent sept à huit fonctionnalités sur les quinze étudiées. Ils proposent tous les trois l'opérateur AND par défaut, les opérateurs + et - ainsi que la formulation d'une équation de recherche par une expression. Les autres options proposées par ces trois moteurs sont le respect des accents (tous sauf Google), l'utilisation de l'opérateur $O R$ (tous sauf $L y c o s$ ) et l'utilisation des parenthèses (permise uniquement par Lycos.)

\section{Caractéristiques des moteurs spécialisés}

En ce qui concerne les trois outils spécialisés dans la recherche d'image, Ditto et Visoo, permettent d'effectuer une requête en utilisant plusieurs mots clés reliés par l'opérateur implicite OR. Ditto ne propose aucune autre fonctionnalité, tandis que Visoo ne propose en plus que l'utilisation des opérateurs arithmétiques + et -.

Il faut noter que les options de recherche proposées par Ditto semblent avoir changé assez fréquemment, dans le sens d'un amoindrissement de ses possibilités. Au début de l'année 2002, par exemple, l'opérateur par défaut était AND, il était possible d'effectuer une recherche par expression, ainsi que de relancer une recherche par l'intermédiaire de mots clés fournis par le moteur, ce qui n'est plus le cas actuellement. De même, dans un article relatant des investigations menées sur différents outils de recherche d'images en juin 2000 [9], il apparaît que Ditto proposait à ce moment-là une page de recherche avancée, et permettait d'utiliser l'opérateur de proximité NEAR ainsi que de limiter la recherche par la date de parution des images.

Le dernier des outils spécialisés dans la recherche d'images, Picsearch, propose six des quinze fonctionnalités recensées, ce qui ne le place pas très loin derrière les trois moteurs généralistes AllTheWeb, Google et Lycos. Picsearch ne permet d'effectuer aucune limitation de recherche par nom de domaine, site ou autre, ni de faire une recherche sur des champs particuliers. Ces fonctionnalités semblent être propres aux moteurs généralistes, car héritées de la recherche de pages web.

\section{La fonction de filtrage}

$\mathrm{Vu}$ l'importance que revêtent les filtres de contenu pour la recherche d'images, il semble intéressant de noter que tous les outils étudiés permettent d'appliquer aux résultats un filtre de contenu adulte. Si cette possibilité est utilisée par tous les moteurs, tous ne la proposent pas de la même manière. Sur Ditto et Picsearch, le filtre est appliqué avant l'indexation, ce qui signifie qu'en principe aucune image à caractère pornographique ou violent ne figure dans l'index de ces moteurs. Sur les autres, l'utilisateur peut choisir d'appliquer ou non ce filtre à sa recherche. Sur AllTheWeb, Altavista, Google et Visoo, le filtre est assez logiquement activé par défaut, alors qu'il est désactivé par défaut sur $L y c o s$, ce qui semble assez surprenant. À noter que seuls Altavista et Lycos permettent de verrouiller le filtre par un mot de passe.

Il faut également préciser que ces filtres sont en réalité loin d'être totalement fiables. En effet, pour qu'une image soit considérée comme susceptible de comporter un contenu choquant, il faut que les mots qui l'accompagnent et grâce auxquels elle est indexée soient répertoriés dans une liste de termes reconnus comme tels. Il suffit donc par exemple que le nom de fichier d'une image n'ait rien à voir avec son contenu ou que les différents termes qui entourent l'image soient neutres pour qu'une image ne soit pas considérée par le moteur comme inappropriée. De ce fait, tous les moteurs avertissent leur public de cette faillibilité et certains, notamment Ditto et 
Picsearch, permettent aux utilisateurs d'être prévenus qu'une image choquante est présente dans leur index par le biais d'un formulaire prévu à cet effet.

\section{Comparaison entre moteurs généralistes et spécialisés}

Si l'on compare globalement les fonctionnalités des outils généralistes par rapport à celles des outils spécialisés dans l'image, il apparaît que les premiers proposent en moyenne un peu moins de neuf fonctionnalités sur les quinze étudiées contre plus de quatre pour les seconds. La figure 1 présente, en outre, le pourcentage d'outils de recherche généralistes (en bleu) et spécialisés (en violet) qui proposent chacune des quinze fonctionnalités étudiées.

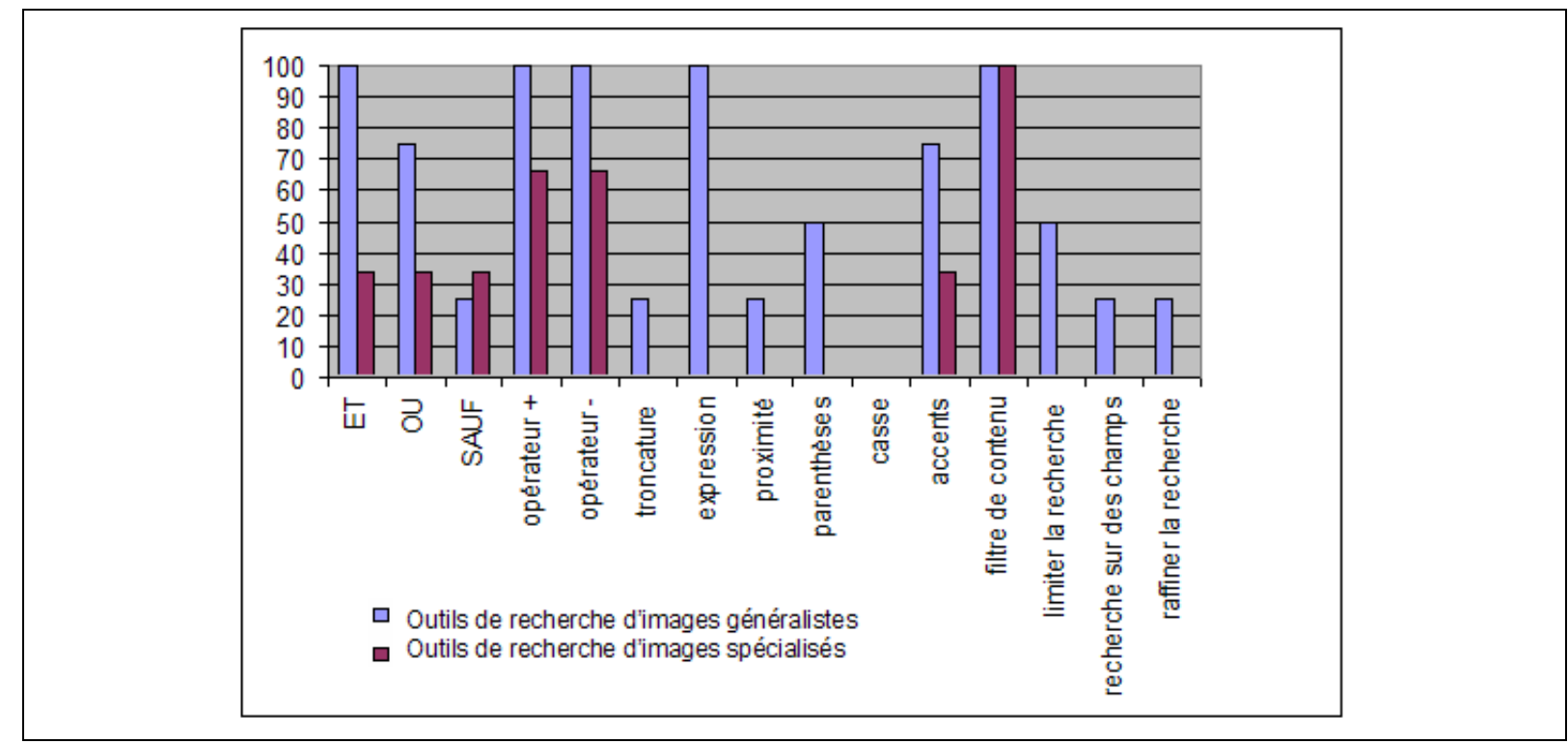

Figure 1: Pourcentage d'outils de recherche généralistes (en bleu) et spécialisés (en violet) qui proposent chacune des 15 fonctionnalités étudiées.

Cette figure fait apparaître assez clairement que les outils généralistes sont, hormis pour I'opérateur SAUF et le filtre de contenu, en proportion toujours plus nombreux à proposer chacune des quinze fonctionnalités étudiées. Certaines de celles-ci, comme la recherche d'expression, sont d'ailleurs proposées uniquement par les quatre outils de recherche généralistes.

D'une manière plus générale, les résultats de ces comparaisons font apparaître que les moteurs spécialisés dans la recherche d'images offrent sur le plan de la syntaxe d'interrogation des possibilités nettement moins étendues que les moteurs dérivant des outils de recherche de pages web. Une des explications possibles de cette différence est que ces derniers ont bénéficié indirectement des développements et efforts déployés dans le cadre de la recherche de pages web (tableau 2). 


\begin{tabular}{|c|c|c|c|c|c|c|c|c|}
\hline & AllTheWeb & Altavista & Google & Lycos & AllTheWeb & Altavista & Google & Lycos \\
\hline & \multicolumn{4}{|c|}{$\begin{array}{l}\text { Interface de recherche de pages } \\
\text { web }\end{array}$} & \multicolumn{4}{|c|}{ Interface de recherche d'images } \\
\hline $\begin{array}{l}\text { opérateur par } \\
\text { défaut }\end{array}$ & AND & OR & AND & AND & AND & OR & AND & AND \\
\hline ET & défaut & O & défaut & défaut & défaut & $\mathrm{O}$ & défaut & défaut \\
\hline OU & 0 & défaut & $\mathrm{O}$ & $\mathrm{N}$ & $\mathrm{O}$ & défaut & $\mathrm{O}$ & $\mathrm{N}$ \\
\hline SAUF & $\mathrm{N}$ & $\mathrm{O}$ & $\mathrm{N}$ & $\mathrm{N}$ & $\mathrm{N}$ & $\mathrm{O}$ & $\mathrm{N}$ & $\mathrm{N}$ \\
\hline opérateur + & $\mathrm{O}$ & $\mathrm{O}$ & $\mathrm{O}$ & $\mathrm{O}$ & $\mathrm{O}$ & $\mathrm{O}$ & $\mathrm{O}$ & $\mathrm{O}$ \\
\hline opérateur - & $\mathrm{O}$ & $\mathrm{O}$ & $\mathrm{O}$ & $\mathrm{O}$ & $\mathrm{O}$ & $\mathrm{O}$ & $\mathrm{O}$ & $\mathrm{O}$ \\
\hline troncature & $\mathrm{N}$ & 0 & $\mathrm{~N}$ & $\mathrm{~N}$ & $\mathrm{~N}$ & 0 & $\mathrm{~N}$ & $\mathrm{~N}$ \\
\hline expression & 0 & 0 & 0 & 0 & 0 & 0 & $\mathrm{O}$ & $\mathrm{O}$ \\
\hline proximité & $\mathrm{N}$ & 0 & $\mathrm{~N}$ & $\mathrm{~N}$ & $\mathrm{~N}$ & 0 & $\mathrm{~N}$ & $\mathrm{~N}$ \\
\hline parenthèses & 0 & 0 & $\mathrm{~N}$ & 0 & $\mathrm{~N}$ & O & $\mathrm{N}$ & 0 \\
\hline casse & $\mathrm{N}$ & $\mathrm{N}$ & $\mathrm{N}$ & $\mathrm{N}$ & $\mathrm{N}$ & $\mathrm{N}$ & $\mathrm{N}$ & $\mathrm{N}$ \\
\hline accents & 0 & 0 & $\mathrm{~N}$ & 0 & 0 & 0 & $\mathrm{~N}$ & 0 \\
\hline $\begin{array}{ll}\text { filtre } \\
\text { contenu }\end{array}$ & $\begin{array}{l}\text { Opar } \\
\text { défaut }\end{array}$ & $\begin{array}{c}\mathrm{O} \text { à } \\
\text { installer }\end{array}$ & $\begin{array}{l}\text { O à } \\
\text { installer }\end{array}$ & $\begin{array}{c}\mathrm{O} \text { à } \\
\text { installer }\end{array}$ & O par défaut & O par défaut & $\begin{array}{l}\text { Opar } \\
\text { défaut }\end{array}$ & $\begin{array}{c}\mathrm{O} \text { à } \\
\text { installer }\end{array}$ \\
\hline $\begin{array}{|ll|}\text { limiter } & \text { la } \\
\text { recherche }\end{array}$ & $\begin{array}{c}\text { langue, } \\
\text { nom de } \\
\text { domaine }\end{array}$ & $\begin{array}{c}\text { langue, } \\
\text { nom de } \\
\text { domaine, } \\
\text { date, } \\
\text { host, } \\
\text { anchor }\end{array}$ & $\begin{array}{c}\text { langue, } \\
\text { nom de } \\
\text { domaine, } \\
\text { date, } \\
\text { formats de } \\
\text { fichier }\end{array}$ & $\begin{array}{l}\text { langue, } \\
\text { nom de } \\
\text { domaine }\end{array}$ & $\mathrm{N}$ & $\begin{array}{c}\text { nom de } \\
\text { domaine, } \\
\text { host }\end{array}$ & $\begin{array}{c}\text { nom de } \\
\text { domaine, } \\
\text { site }\end{array}$ & $N$ \\
\hline \begin{tabular}{|l|} 
recherche sur \\
des champs
\end{tabular} & $\begin{array}{l}\text { titre, url, } \\
\text { liens, }, .\end{array}$ & \begin{tabular}{|c|} 
titre, url, \\
liens, \\
texte, \\
nom de \\
fichier de \\
l'image, \\
..
\end{tabular} & $\begin{array}{l}\text { titre, url, } \\
\text { liens, } \\
\text { texte,... }\end{array}$ & $\begin{array}{l}\text { titre, url, } \\
\text { liens, }, . .\end{array}$ & $\mathrm{N}$ & title, url & $\mathrm{N}$ & $\mathrm{N}$ \\
\hline $\begin{array}{ll}\text { raffiner } & \text { la } \\
\text { recherche }\end{array}$ & $\mathrm{N}$ & $N$ & 0 & $\mathrm{~N}$ & $\mathrm{~N}$ & O & $\mathrm{N}$ & $\mathrm{N}$ \\
\hline Total & 10 & 13 & 9 & 9 & 7 & 13 & 8 & 7 \\
\hline Moyenne & \multicolumn{4}{|c|}{10,25} & \multicolumn{4}{|c|}{8,75} \\
\hline
\end{tabular}

Tableau 2: Fonctionnalités des moteurs de recherche de pages web et leurs correspondants pour la recherche d'images. $\mathrm{N}$ : fonctionnalité non disponible pour l'outil considéré ; $O$ : fonctionnalité disponible pour l'outil considéré. 
La comparaison des fonctionnalités syntaxiques des outils de recherche de pages web et de leurs équivalents pour la recherche d'images fait apparaître une similitude forte entre les possibilités proposées par ces différentes interfaces.

Il semble tout d'abord que les interfaces de recherche d'images des moteurs généralistes proposent légèrement moins de possibilités que leurs interfaces de recherche textuelle (en moyenne un peu moins de neuf pour les interfaces de recherche d'images contre plus de dix pour les interfaces de recherche textuelle). Cette perte s'effectue principalement sur la possibilité de limiter sa recherche par des éléments tels que la langue, la date, le nom de domaine, et pour la possibilité d'effectuer une recherche sur des champs particuliers. Cette dernière option, qui est disponible sur les quatre moteurs de recherche de texte, ne l'est pour la recherche d'images que sur Altavista. Elle permet de rechercher des images en spécifiant un mot qui doit apparaître dans le titre ou dans I'URL de la page.

En ce qui concerne la limitation de la recherche par certains critères, seuls deux moteurs généralistes sur les quatre proposent d'en effectuer une. Altavista permet de rechercher des images sur une adresse ou un domaine spécifiques. Mais il est pour cela nécessaire d'employer une syntaxe précise qui n'est indiquée que dans l'aide du site français. Il s'agit en fait de la même syntaxe que lors d'une recherche de texte, mais l'aide du site principal n'indique pas dans sa rubrique « images » que ces options sont disponibles. Google permet de limiter la recherche à un site ou un domaine précis. Dans la page de recherche avancée, un cadre est réservé à cette option; il n'est donc pas nécessaire d'employer une syntaxe particulière.

Aucun des quatre moteurs de recherche de pages web n'a transposé à la recherche d'images la possibilité de limiter la recherche par la langue ou la date. S'il semble en effet peu intéressant d'utiliser la langue pour rechercher des images, la disparition de la possibilité de rechercher par date peut sembler par contre plus surprenante. Si l'on écarte ces deux groupes d'éléments qui témoignent d'une perte de fonctionnalités entre les moteurs de recherche de texte et leur interface de recherche d'images, on constate que les autres possibilités d'interrogation sont conservées. Une seule différence est observée, sur Al/TheWeb, qui ne prend pas en compte les équations contenant des parenthèses dans l'interface image, alors qu'on peut les employer dans l'interface de recherche de pages web.

\section{Critères de recherche propres aux images}

Il s'agit ici d'examiner les possibilités offertes par les différents outils concernant les critères de recherche propres aux images. Ces critères concernent la taille, le poids et le format des images. Ils peuvent également prendre en compte le type d'image (dessin, photographie) ou le fait que l'image est en couleurs ou en noir et blanc.

Le tableau 3 montre que seulement deux moteurs permettent de sélectionner la taille des images recherchées. Il s'agit de Google et de Picsearch, qui proposent tous les deux de sélectionner une taille d'image parmi six (« very small, small, medium... »). Il n'y a que trois moteurs (AllTheWeb, Google et Visoo) qui proposent de sélectionner le format des images retrouvées : formats GIF (Graphics Interchange Format), JPEG (Joint Photographic Experts'Group) et BMP. Il semble assez surprenant que le format BMP soit disponible (format qui n'est pas propre aux pages web), alors que le format PNG (Portable Network Graphics) qui est, lui, un format propre à ces pages, ne l'est pas. 


\begin{tabular}{|c|c|c|c|c|c|c|c|}
\hline & AllTheWeb & Altavista & Google & Lycos & Ditto & Picsearch & Visoo \\
\hline taille/poids & $\mathrm{N}$ & $\mathrm{N}$ & $\begin{array}{c}\text { O } \\
6 \text { tailles } \\
\text { possibles }\end{array}$ & $\mathrm{N}$ & $\mathrm{N}$ & $\begin{array}{c}0 \\
6 \text { tailles } \\
\text { possibles }\end{array}$ & $\mathrm{N}$ \\
\hline format & $\begin{array}{c}\mathrm{O} \\
\text { (jpg,gif,bmp) }\end{array}$ & $\mathrm{N}$ & $\begin{array}{c}\mathrm{O} \\
\text { (jpg, gif) }\end{array}$ & $\mathrm{N}$ & $\mathrm{N}$ & $\mathrm{N}$ & $\begin{array}{c}\text { O } \\
\text { (jpg, gif, } \\
\text { bmp) }\end{array}$ \\
\hline type & $\begin{array}{c}\mathrm{O} \\
\text { Line art }\end{array}$ & $\begin{array}{c}\mathrm{O} \\
\text { (photos, } \\
\text { dessins, } \\
\text { boutons, } \\
\text { bannières) }\end{array}$ & $\mathrm{N}$ & N & $\mathrm{N}$ & $\begin{array}{c}\mathrm{O} \\
\text { (images ou } \\
\text { animations) }\end{array}$ & $\begin{array}{l}\mathrm{O} \text { (photos, } \\
\text { bannières) }\end{array}$ \\
\hline $\begin{array}{l}\text { couleur/noir } \\
\text { et blanc }\end{array}$ & $\mathrm{O}$ & $\mathrm{O}$ & $\mathrm{O}$ & $\mathrm{N}$ & $\mathrm{N}$ & $\mathrm{O}$ & $\mathrm{O}$ \\
\hline autres & transparence & & & & & & $\begin{array}{c}\text { recherche de } \\
\text { texte dans } \\
\text { les images + } \\
\text { recherche } \\
\text { spécifique } \\
\text { sur le nom } \\
\text { de fichier }\end{array}$ \\
\hline Total & 3 & 2 & 3 & 0 & 0 & 3 & 3 \\
\hline Moyenne & \multicolumn{4}{|c|}{2} & \multicolumn{3}{|c|}{2} \\
\hline
\end{tabular}

Tableau 3: critères de recherche propres aux images des sept outils de recherche étudiés. . N : fonctionnalité non disponible pour l'outil considéré: $O$ : fonctionnalité disponible pour l'outil considéré.

Davantage d'outils proposent de spécifier des critères relatifs au type de l'image ou à sa catégorie de couleurs. Ainsi, quatre outils proposent de restreindre la recherche à un certain type d'images, mais cette catégorie est en fait très hétérogène selon les cas. Le plus souvent, il s'agit de sélectionner des photographies, des dessins ou des images « line art» (images binaires à deux couleurs, une pour le fond et une pour les objets). Altavista propose aussi de trouver des boutons et des bannières. Picsearch, enfin, propose de choisir entre des images ou des animations. Cinq outils permettent par ailleurs de préciser si les résultats doivent être en couleurs ou en noir et blanc.

Alltheweb, Google, Picsearch et Visoo, en proposant respectivement trois critères de recherche spécifiques à l'image, sont les outils qui proposent le plus de possibilités.

Avec deux critères possibles, Altavistan'est plus ici l'outil qui offre le plus de fonctionnalités.

Lycos et Ditto, enfin, ne proposent aucune possibilité de préciser la recherche avec des caractéristiques propres aux images. Visoo est le seul à proposer une recherche de texte apparaissant dans les images (méthode basée sur les principes de la reconnaissance optique de caractères) qui permet de retrouver des images contenant un texte particulier. Enfin, il est particulièrement frappant de voir que Ditto, dont les fonctionnalités syntaxiques étaient très limitées, n'offre en retour aucune possibilité de recherche sur des critères propres aux images.

Si l'on compare globalement les fonctionnalités des outils généralistes par rapport à ceux qui sont spécialisés dans l'image, les premiers comme les seconds proposent en moyenne deux 
fonctionnalités sur les quatre étudiées (tableau 3). Globalement, la différence qui apparaissait sur le plan des possibilités syntaxiques d'interrogation entre outils généralistes et outils spécialisés a donc tendance à s'atténuer pour les critères propres à la recherche d'images.

\section{Présentation des résultats}

La manière dont les résultats d'une recherche d'images sont présentés à l'utilisateur conditionne en grande partie le confort de celui-ci. L'information qui accompagne chaque image et qui concerne à la fois l'image elle-même (sa taille, son format, etc.) et sa source (de quel site elle est issue? que peut-on savoir sur ce site avant d'y accéder ? etc.), ainsi que les modalités d'accès à la source de l'image, sont également des critères importants. L'ensemble de ces différents éléments est présenté dans le tableau 4.

\begin{tabular}{|l|c|c|c|c|c|c|c|}
\hline \multicolumn{2}{|c|}{ AllTheWeb } & Altavista & Google & Lycos & Ditto & Picsearch & Visoo \\
\hline $\begin{array}{l}\text { nombre de } \\
\text { vignettes par } \\
\text { page }\end{array}$ & 9 & 15 & 20 & 9 & 12 & 16 & 15 \\
\hline $\begin{array}{l}\text { Informations } \\
\text { sur l'image }\end{array}$ & $\begin{array}{c}\text { poids, } \\
\text { taille, } \\
\text { format, } \\
\text { nom de } \\
\text { fichier }\end{array}$ & poids, taille & poids, taille & poids, taille & $\begin{array}{c}\text { poids, taille, } \\
\text { nom de } \\
\text { fichier }\end{array}$ & $\begin{array}{c}\text { poids, taille, } \\
\text { nombre de } \\
\text { couleurs, } \\
\text { format, nom } \\
\text { de fichier }\end{array}$ & poids, taille \\
\hline $\begin{array}{l}\text { informations } \\
\text { sur la page }\end{array}$ & $\mathrm{N}$ & URL & URL & URL & URL, titre & URL & URL \\
\hline $\begin{array}{l}\text { lien vers } \\
\text { page/image }\end{array}$ & $\begin{array}{c}\text { les 2 au } \\
\text { choix }\end{array}$ & page & $\begin{array}{c}\text { les 2 au } \\
\text { choix }\end{array}$ & $\begin{array}{c}\text { dépend de } \\
\text { la source de } \\
\text { l'image }\end{array}$ & page & $\begin{array}{c}\text { les 2 au } \\
\text { choix }\end{array}$ & $\begin{array}{c}\text { les } 2 \text { au } \\
\text { choix }\end{array}$ \\
\hline $\begin{array}{l}\text { copyright } \\
\text { explicite }\end{array}$ & O & O & O & O & O & O & $\mathrm{N}$ \\
\hline
\end{tabular}

Tableau 4 : Présentation des résultats des sept outils étudiés

L'ensemble des sept outils étudiés propose des vignettes dans la page de résultats, c'est-à-dire des versions réduites de l'image d'origine. Le nombre de vignettes présentées par page varie de neuf pour Lycos à vingt pour Google. En ce qui concerne les informations sur les images qui figurent sur la page des résultats, le tableau 4 montre que tous les outils indiquent la taille des images (en pixels) et leur poids (en kilo-octets). Si globalement beaucoup d'informations concernant les images sont disponibles, particulièrement sur AllTheWeb, Altavista, Ditto et Picsearch, très peu d'informations concernant le site d'où elles proviennent sont généralement fournies. Concernant l'accès aux images, la plupart des outils proposent à la fois un lien direct vers l'image et un lien vers la page web où elle apparaît.

Enfin, tous les outils, excepté Visoo, mentionnent l'existence de droits d'exploitation attachés aux images auxquelles ils donnent accès ainsi que l'obligation de demander à leurs propriétaires l'autorisation de les utiliser pour un usage autre que personnel. 


\section{Discussion et conclusions}

Cette étude avait pour objectif de produire une évaluation comparative des fonctionnalités de différents outils de recherche d'images sur Internet. En premier lieu, les résultats présentés ici mettent en évidence une certaine hétérogénéité des outils étudiés, tant du point de vue des possibilités de syntaxe d'interrogation que du point de vue des possibilités qu'ils offrent de recherche propres aux images. Il faut noter que cette hétérogénéité renforce les difficultés qu'éprouvent généralement les utilisateurs d'outils de recherche sur Internet, en leur imposant de fait un perpétuel effort d'adaptation lorsqu'ils souhaitent passer d'un outil à un autre.

En second lieu et de façon assez surprenante, les outils qui ont été spécifiquement développés pour la recherche des images (Ditto, Picsearch, Visoo) ne s'avèrent globalement pas être les plus performants. Cette situation semble provenir du fait que les outils de recherche d'images qui dérivent directement des outils de recherche de pages web (Alltheweb, Altavista, Google et Lycos) sont, en raison de leur conception, très proches de ces outils, et ont, par conséquent, bénéficié indirectement des moyens importants mis en place pour les développer et les améliorer. Ces moyens n'ont visiblement pas été mis en place pour les outils spécifiquement développés pour la recherche des images, ce qui fait qu'ils sont globalement moins complets, surtout en ce qui concerne leur syntaxe d'interrogation, même si les possibilités qu'ils offrent de recherche spécifiques aux images se révèlent relativement proches de celles proposées par les outils dérivant des outils de recherche de pages web.

Il faut noter que ce paradoxe existe également pour les outils de recherche de pages web dans le domaine de la bio-médecine où, de même, les outils spécialisés s'avèrent globalement moins complets que les outils généralistes [34]. Dans les deux cas, il est surprenant que des outils spécifiquement développés pour rechercher des informations dans un domaine particulier ou des éléments spécifiques comme les images soient moins performants que des outils généralistes. II semble pourtant qu'on est en droit d'attendre d'outils spécialement dédiés à un type de recherche une performance au moins égale à celle des outils généralistes, ce qui n'est visiblement pas toujours le cas.

En conclusion, si les outils étudiés dans cet article souhaitent fournir aux usagers d'Internet des services de plus en plus performants, il semble nécessaire qu'ils continuent d'évoluer, non seulement afin de proposer des fonctionnalités plus abouties et en plus grand nombre, mais également pour faire face au nombre grandissant d'images disponibles sur Internet. Les pistes à suivre pour que l'évolution fonctionnelle de ces outils soit réelle et bénéfique aux utilisateurs passent très certainement par la généralisation de l'utilisation des métadonnées associées aux images présentes sur les sites web, mais également par l'utilisation combinée des méthodes de recherche par le texte et par l'image $[24,35,36]$. Enfin, vu le caractère très concurrentiel de l'espace occupé par ces outils de recherche, il est probable que cette évolution soit accompagnée par un certain nombre d'absorption d'outils par d'autres, ou de cessation d'activité, comme cela a été récemment le cas de Diggitet Visoo.

FEVRIER 2004 


\section{Références}

[1] Lawrence S., Giles CL. Accessibility of information on the web. Nature, $1999 ; 400: 107-109$.

[2] Winship IR. World Wide Web searching tools - an evaluation. Vine, 1995 ; 99 : 49-54.

[3] Chu HT., Rosenthal M. Search engines for the World Wide Web: a comparative study and evaluation methodology. Proc ASIS Annu Meet, $1996 ; 33: 127-135$.

[4] Dong $X$., Su L. Search engines on the world wide web and information retrieval on the Internet: a review and evaluation. Online \& CD ROM Review, $1997 ; 21: 67-81$.

[5] Xie M., Wang H., Goh TN. Quality dimensions of Internet search engines. Journal of Information Science, $1998 ; 24: 365-372$.

[6] Wang H., Xie M., Goh TN. Service quality of Internet search engines. Journal of Information Science, $1999 ; 25: 499-507$.

[7] Oppenheim C., Morris A., McKnight $C$. The evaluation of WWW search engines. Journal of documentation, $2000 ; 56: 190-211$.

[8] Moulis A.-M. La recherche d'images sur Internet. Documentaliste - Sciences de l'information, $1999 ; 36$ (3) : 179-186.

[9] Hassan I., Zhang J. Image searching feature analysis. Online Information Review, $2001 ; 25$

(2) : 103-114.

[10] Pack T. Image searching across the web. Online, $2002 ; 26$ (1) : 30-34.

[11] Berinstein P. Turning visual: image search engines on the Web. Online, 1998 ; may- june : 3742.

[12] Lettre « Recherche et Référencement». Avril 2003. 〈http://www.abondance.com〉

[13] Lettre «Recherche et Référencement ». Mai 2003. 〈http://www.abondance.com»

[14] Lettre « Recherche et Référencement ». Juin 2003. 〈http://www.abondance.com>

[15] Boudry $C$. En savoir plus sur les images numériques. Module « Outils de recherche d'images sur Internet». 〈www.ccr.jussieu.fr/urfist/image_numerique/Image_numerique1.htm〉 (au 21 février 2004).

[16] Collard C., Giannatasio I., Melot M. Les images dans les bibliothèques. Paris: Cercle de la Librairie, 1995.

[17] Kattnig C. Gestion et diffusion d'un fonds d'image. Paris: ADBS, Nathan, 2002. (Nathan Université. Coll. 128, série Information, documentation).

[18] Blery G. La mémoire photographique : étude de la classification des images et analyse de leur contenu. Thèse de troisième cycle, Strasbourg, université Louis Pasteur, 1976.

[19] Jacobs C. If a picture is worth a thousand words, then. The Indexer, $1999 ; 21$ (3) : 112-123.

[20] Furner J. Digital images in libraries : an overview. Vine, 1998 ; 107 : 3-7.

[21] Rasmussen EM. Indexing images. Annual Review of Information Science and Technology, $1997: 32: 169-196$. 
[22] Markey K. Interindexer consistency tests: a literature review and report of a test of consistency in indexing visual materials. Library and Information Science Research, 1984 ; 6 (2): 155-177.

[23] Enser PGB. Progress in Documentation. Pictorial Information Retrieval, 1995 ; 51 (2) : 126170.

[24] Eakins J. Techniques for image retrieval. Library and Information Briefings, 1998 ; 85 : 115.

[25] Kato T. Database architecture for content-based image retrieval. In: Image Storage and Retrieval Systems (Jambardino A.A. and Niblack W.R. Eds) 1992 ; Proc SPIE 1662 : 112-123.

[26] Coster M., Chermant JL. Précis d'analyse d'images. Paris : Les Presses du CNRS, 1989.

[27] Eakins J., Graham ME. Content-based Image Retrieval: JISC Technology Application Programme Report 39. 〈http://www.unn.ac.uk/iidr/research/cbir/report.html> (au 21 février 2004).

[28] Nastar C. Indexation et recherche d'images: enjeux, méthodes et perspectives. Actes du Congrès IDT 1999, Paris, France.

[29] Boudry C. Classification cellulaire par morphologie mathématique. Thèse de Doctorat, Caen, université de Caen, 1997.

[30] Pentland A., Picard R.W., Sclaroff S. Photobook: tools for content-based manipulation of image databases. International Journal of Computer Vision, 1996 ; 18 (3) : 233-254.

[31] Eakins J., Boarman JM., Graham ME. Similarity retrieval of trademark images. IEEE Multimedia, $1998 ; 5$ (2) : 53-63.

[32] Smith JR., Chang SF. An Image and Video Search Engine for the World-Wide Web. Proc. IS\&T/SPIE Storage \& Retrieval for Still Image and Video Databases V, San Jose, CA, 1997 : 84-95.

[33] Lu G., Williams B. You C. An effective World Wide Web image search engine. Journal of Information Science, $2001 ; 27$ (1) : 27-37.

[34] Boudry $C$. Evaluation of Web search engine performances in the field of biology. MédecineSciences, $2002 ; 18: 1107-1112$.

[35] Enser P. Visual image retrieval: seeking the alliance of concept-based and content-based paradigms. Journal of Information Science, $2000 ; 26$ (4) : 199-210.

[36] Day M. Metadata. Image retrieval: combining content-based and metadata-based approches. Adriane, 1999. 〈www.ariadne.ac.uk/issue19/metadata/intro.html> 